\title{
Ocular Changes -Cataract And Retinal Lesion- In Spontaneously Diabetic Torii (SDT) Fatty Rats, An Obese Type 2 Diabetic Model
}

\author{
Tatsuya Maekawa ${ }^{1}$, Sanae Nakamura ${ }^{2}$, Katsuhiro Miyajima ${ }^{3}$, Kinuko Uno ${ }^{3}$, Ayane Yamaguchi ${ }^{3}$, Kouhei \\ Mandai $^{3}$, Takayuki Gotoh ${ }^{4}$, Masami Shinohara ${ }^{4}$, Yuichi Shinozaki ${ }^{1}$, Tomohiko Sasase ${ }^{1}$, Fatchiyah \\ Fatchiyah $^{5}$, and Takeshi Ohta ${ }^{1}$ \\ ${ }^{1}$ Laboratory of Animal Physiology and Functional Anatomy, Graduate School of Agriculture, Kyoto University \\ Kitashirakawa Oiwake-cho, Sakyo-ku, Kyoto 606-8502, Japan \\ ${ }^{2}$ JT Creative Service co., ltd., Takatsuki office \\ 1-1, Murasaki-cho, Takatsuki, Osaka, 569-1125, Japan \\ ${ }^{3}$ Department of Nutritional Science and Food Safety Faculty of Applied Biosciences, Tokyo University of Agriculture \\ 1-1-1, Sakuragaoka, Setagaya-ku, Tokyo, 156-8602, Japan \\ ${ }^{4}$ Clea Japan Inc. \\ 1-2-7, Higashiyama, Meguro-ku, Tokyo 153-8533, Japan \\ ${ }^{5}$ Research Center for Smart Molecules of Natural Genetics Resources (SMONAGENES) Brawijaya University \\ Jl. Veteran, Ketawanggede, Kec. Lowokwaru, Kota Malang, Jawa Timur 65145, Indonesia \\ * Corresponding author: Takeshi Ohta; e-mail: ota.takeshi.3a@ kyoto-u.ac.jp; tel.: +81-75-753-6325
}

\begin{abstract}
Cataract and retinopathy remain the preventable cause of blindness worldwide, and many pharmacological strategies have been proposed for the treatment of these eye diseases. Animal models play an important role in understanding the pathophysiological features of eye disease and developing for a new therapy. In this study, we investigated the development of cataract and retinal lesion with diabetes using an obese type 2 diabetic models SDT fatty rat. Macroscopic analysis in eyes was performed from 16 to 24 weeks of age and histological analysis was performed at 24 weeks of age. As a result, the lens cloudiness was observed from 19 weeks of age and the degree of the cloudiness was more progressed until 24 weeks of age. Histopathological findings, such as degeneration of lens fiber and shortening and irregular arrangement of cone and rod in retinal tissue, were observed at 24 weeks of age. In conclusion, SDT fatty rats may be useful to understand the pathological features in diabetic cataract and retinopathy develop a new therapy for the disease.
\end{abstract}

Keywords: Cataract, Retinopathy, Diabetes, SDT fatty rat

\section{INTRODUCTION}

Cataract and retinopathy are an ocular lesion which leads to visual disability and blindness [1-3]. It is estimated that 191 million people are visually disabled all over the world, and cataract contributes to $50 \%$ of blindness [1], [4]. The prevalence of cataracts increases after the age of 40 years, ranging from $3.9 \%$ in the 55-64 years age group to $92.6 \%$ in the age group 80 years and older [5-7]. Cataracts are caused by lens degradation characterized by clouding with blurry or hazy vision, and apart from aging, smoking, ultraviolet radiation (UV), gender, steroids, diabetes, and high body mass index are responsible for the development of cataract [1], [8]. Currently, the only cure is surgical removal of the cataract lenses and replacing them with new lenses made of synthetic polymers, and the incidence of cataract is so large that the surgical facilities are unable to respond to the problem. Therefore, pharmacological interventions that maintain the transparency and function of lens is desired.

On the other hand, the main cause of retinopathy is diabetes, and diabetic retinopathy (DR) is famous as one of the three major diabetic complications [9]. DR also accounts for $2.6 \%$ of all blindness, though not as much as cataracts [10]. Anti-VEGF agents, corticosteroids and non-steroidal anti-inflammatory drugs are used for DR, and laser treatment is used to suppress the progression [11], but there is still no cure for DR.

Animal models play a key role to progress a better understanding of the pathophysiology in various diseases and developing new therapies for the diseases. The Spontaneously Diabetic Torii (SDT) fatty rat, which is formed by inserting the fa allele of the Zucker fatty rat into the SDT rat genome, shows obesity, hyperglycemia, and dyslipidemia from approximately 5-6 weeks of age [12], [13]. With the early incidence of diabetes, diabetes-related complications in SDT fatty rats were observed at younger age compared with the SDT rats [14], [15]. The microvascular complications such as retinopathy, nephropathy, and neuropathy, were observed after 16 weeks of age in SDT fatty rats [16-19].

In this study, we investigated the development of primarily diabetic cataracts in SDT fatty rats by macroscopic and microscopic analyses, and changes in the retina were also evaluated microscopically. 


\section{Animals}

Male SDT fatty rats and Sprague-Dawley (SD) rats (CLEA Japan, Tokyo, Japan) were used for the study $(n=4)$. The SD rats were used as a normal rat. All animal procedures and the protocol complied with the guidelines for animal experimentation set by the Ethics Committee for Animal Use at Kyoto University and Tokyo University of Agriculture. The rats were maintained at $23 \pm 3^{\circ} \mathrm{C}$ on a $12 \mathrm{~h} / 12 \mathrm{~h}$ light-dark cycle with ad libitum access to a standard diet (CE-2; CLEA Japan, Tokyo, Japan) and water.

\section{Measurement of biological parameters}

Body weight and biochemical parameters such as serum glucose, insulin, triglyceride (TG), and total cholesterol (TC) levels, were measured at 24 weeks of age. Blood samples were collected from the tail vein under non-fasted condition. Serum glucose, TG, and TC levels were measured using commercial kits (Roche Diagnostics, Basel, Switzerland) and an automatic analyzer (Hitachi 7180; Hitachi HighTechnologies, Tokyo, Japan). Commercial EnzymeLinked Immuno Sorbent Assay (ELISA) kits were used to measure serum insulin (Rat insulin ELISA Kit; Morinaga Institute of Biological Science, Yokohama, Japan).
Degree of lens cloudiness in all rats was evaluated once a week from 16 to 24 weeks of age. The lens cloudiness was examined macroscopically, and findings were graded from normal (-) to severe (3+). Scoring criteria is shown in Fig. 1.

\section{Microscopy (Histological analysis)}

Necropsy was performed at 24 weeks of age. All animals were sacrificed by exsanguination under isoflurane anesthesia. The eyes were immediately sampled and fixed in modified Davidson's fluid. After resection, the tissue was paraffin-embedded by standard techniques and thin-sectioned ( 3 to $5 \mu \mathrm{m}$ ). The sections were stained with hematoxylin and eosin (HE). Those samples were examined histopathological.

\section{Statistical analysis}

The results of biological parameters were expressed as the mean \pm standard deviation. Statistical analyses of differences between mean values were performed as follow: homogeneity of variance was tested with an F-test followed by Student's $t$-test or Aspin-Welch's $t$-test for homoscedastic data or heteroscedastic data, respectively. All statistical analyses were performed using Statlight 2000 (Yukms Corp., Kawasaki, Japan). Differences were defined as significant at $\mathrm{P}<0.05$.

\section{Observation of the eye}

(A) - : Normal

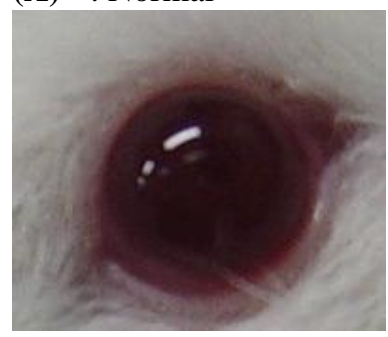

(B) $1+$ : slight

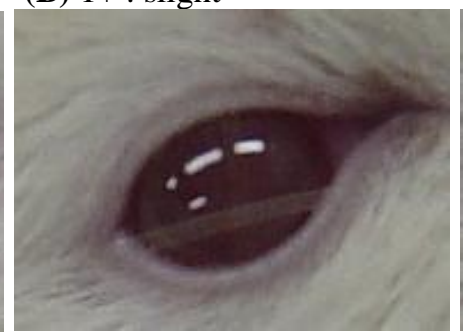

(C) $2+$ : moderate

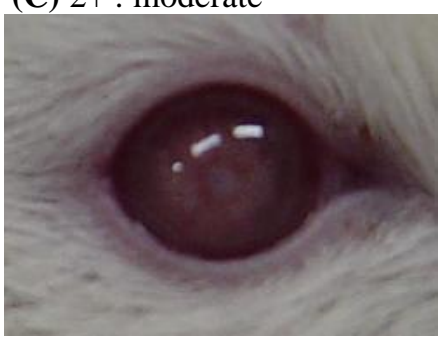

(D) $3+$ : severe

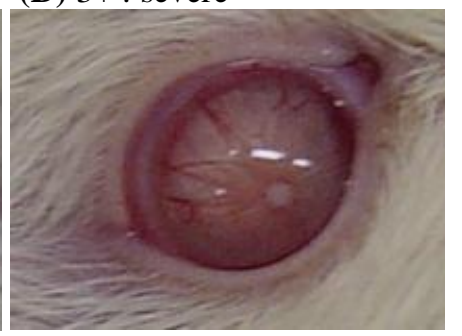

Figure 1. Scoring criteria of lens cloudiness in rats.

Table 1. Biological parameters at 24 weeks of age in SD rats and SDT fatty rats

\begin{tabular}{cccccc}
\hline & $\begin{array}{c}\text { Body weight } \\
(\mathrm{g})\end{array}$ & $\begin{array}{c}\text { Plasma glucose } \\
(\mathrm{mg} / \mathrm{dL})\end{array}$ & $\begin{array}{c}\text { Plasma insulin } \\
(\mathrm{ng} / \mathrm{mL})\end{array}$ & $\begin{array}{c}\text { Plasma TG } \\
(\mathrm{mg} / \mathrm{dL})\end{array}$ & $\begin{array}{c}\text { Plasma TC } \\
(\mathrm{mg} / \mathrm{dL})\end{array}$ \\
\hline SD & $708.6 \pm 22.6$ & $122.5 \pm 6.9$ & $3.6 \pm 1.1$ & $270.6 \pm 67.2$ & $84.3 \pm 18.0$ \\
SDT fatty & $514.9 \pm 52.1^{* *}$ & $899.0 \pm 108.0^{* *}$ & $1.2 \pm 0.6^{* *}$ & $1023.7 \pm 621.1$ & $153.3 \pm 48.7^{*}$ \\
\hline
\end{tabular}

Data represent means \pm standard deviation $(n=4)$.

$* P<0.05, * * P<0.01$ vs. SD rats.

\section{RESULTS AND DISCUSSIONS}

SDT fatty rats at 24 weeks of age showed significant hyperglycemia and hyperlipidemia (Table 1). Moreover, the serum insulin level in SDT fatty rats significantly decreased as compared with that in SD rats (Table 1). SDT fatty rats showed a hyperinsulinemia at 4-8 weeks of age, but the insulin levels dramatically decreased after 16 weeks of age [14]. Genetic weakness of the pancreatic function in SDT fatty rats is considered to induce the rapid decline of the insulin levels. Furthermore, body weight in SDT fatty rats showed a significant decrease as compared with that in SD rats. SDT fatty rats reportedly showed 
an increase of body weight compared with SD rats from 4 to 8 weeks of age, but the body weight in SDT fatty rats decreased after 24 weeks of age [14]. The decrease in body weight of SDT fatty rats may be caused by energy loss in the body with sustained severe diabetic state.

Macroscopic ocular change, lens cloudiness, was observed from 19 weeks of age in SDT fatty rats, but was not seen in SD rats during the observational periods (Table 2). The degree of lens cloudiness in three SDT fatty rats (No.1-3) was more progressed with aging, and the scoring grade was severe $(3+)$ at 24 weeks of age (Table 2). Moreover, histological findings such as degeneration of lens fiber including swelling, fragmentation, Morgagnian globule and liquefaction were observed in SDT fatty rats at 24 weeks of age, but not seen in SD rats (Figure 2). Additionally, shortening and irregular arrangement of cone and rod in the retina were observed in a small number of SDT fatty rats showing severe cataracts, but not in SD rats (Figure 3).

Table 2. Score of lens cloudiness in SD and SDT fatty rats

\begin{tabular}{|c|c|c|c|c|c|c|c|c|}
\hline \multirow{2}{*}{$\begin{array}{l}\text { Strain \& } \\
\text { Individual No. }\end{array}$} & & \multicolumn{7}{|c|}{ Weeks of age } \\
\hline & & 16 to 18 & 19 & 20 & 21 & 22 & 23 & 24 \\
\hline \multicolumn{9}{|l|}{ SD rat } \\
\hline \multirow[t]{2}{*}{ No.1 } & Right & - & - & - & - & - & - & - \\
\hline & Left & - & - & - & - & - & - & - \\
\hline \multirow[t]{2}{*}{ No.2 } & Right & - & - & - & - & - & - & - \\
\hline & Left & - & - & - & - & - & - & - \\
\hline \multirow[t]{2}{*}{ No.3 } & Right & - & - & - & - & - & - & - \\
\hline & Left & - & - & - & - & - & - & - \\
\hline \multirow[t]{2}{*}{ No.4 } & Right & - & - & - & - & - & - & - \\
\hline & Left & - & - & - & - & - & - & - \\
\hline \multicolumn{9}{|l|}{ SDT fatty rat } \\
\hline \multirow[t]{2}{*}{ No.5 } & Right & - & $1+$ & $2+$ & $2+$ & $3+$ & $3+$ & $3+$ \\
\hline & Left & - & - & - & $1+$ & $2+$ & $3+$ & $3+$ \\
\hline \multirow[t]{2}{*}{ No.6 } & Right & - & - & $2+$ & $2+$ & $2+$ & $3+$ & $3+$ \\
\hline & Left & - & - & - & - & - & - & - \\
\hline \multirow[t]{2}{*}{ No.7 } & Right & - & $1+$ & $2+$ & $2+$ & $2+$ & $3+$ & $3+$ \\
\hline & Left & - & - & - & - & $1+$ & $1+$ & $3+$ \\
\hline \multirow[t]{2}{*}{ No.8 } & Right & - & - & - & - & - & - & - \\
\hline & Left & - & - & $1+$ & $1+$ & $1+$ & $1+$ & $1+$ \\
\hline
\end{tabular}

- normal; $1+$ slight; $2+$ moderate; $3+$ severe
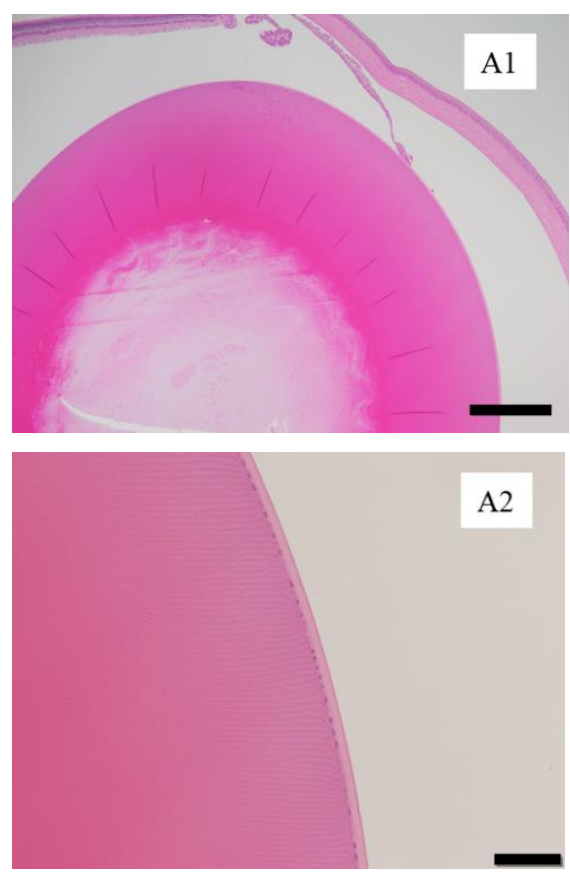
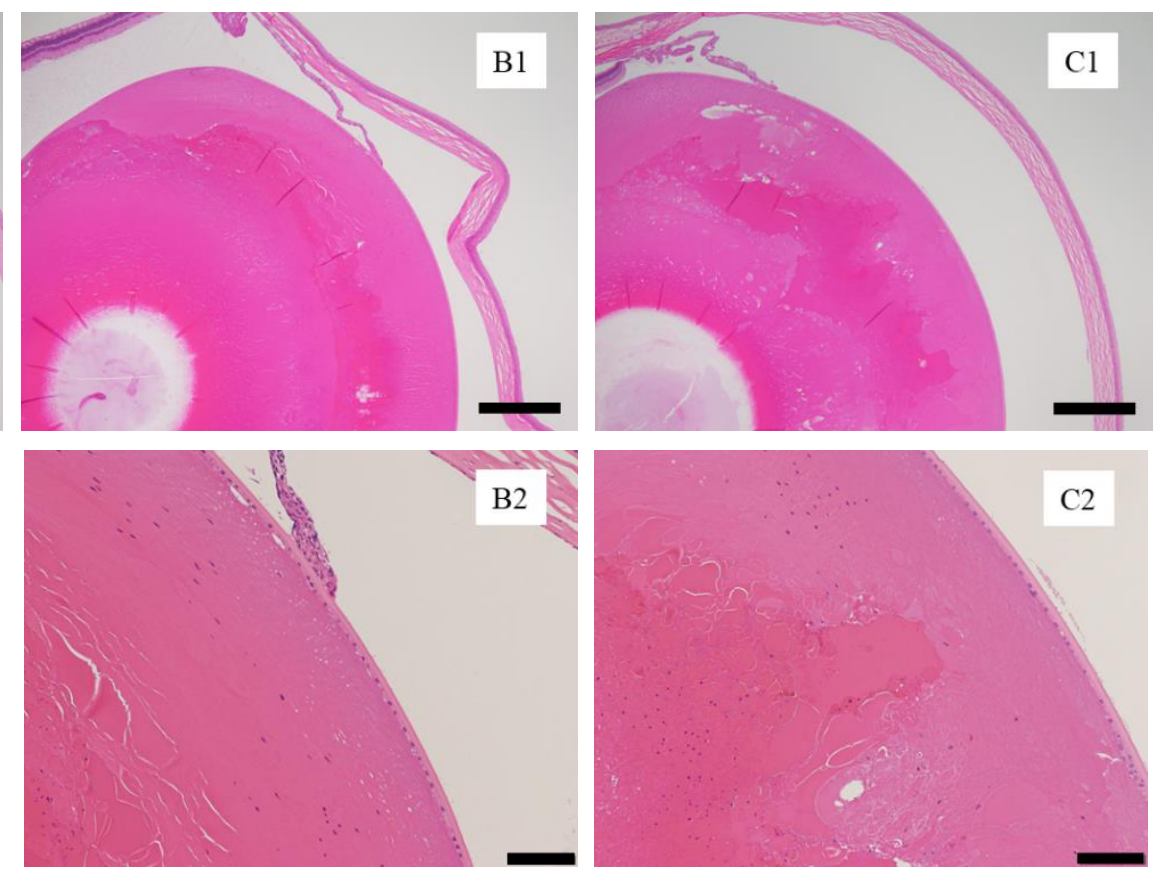

Figure 2. Histopathological observation of lens at 24 weeks of age in SD and SDT fatty rats. A1: Normal lens in SD rats. A2: Higher magnification of A1. B1: Slight cataract in SDT fatty rats. B2: Higher magnification of B1. C1: Severe cataract in SDT fatty rats. C2: Higher magnification of C1. Bar = $200 \mathrm{~mm}$ in A1, B1 and C1, $100 \mathrm{~mm}$ in A2, B2 and $\mathrm{C} 2$, respectively. 

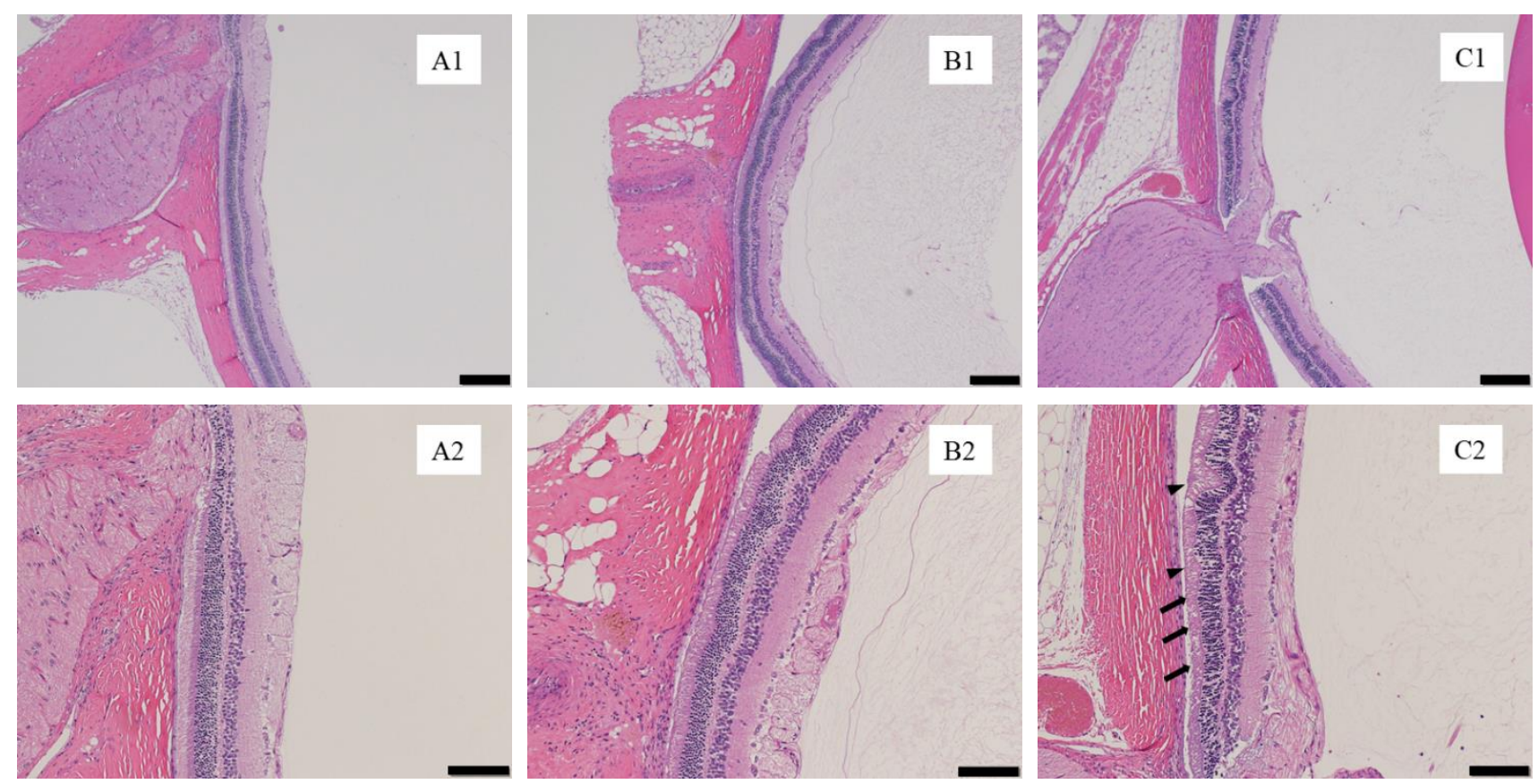

Figure 3. Histopathological observation of retina in SD and SDT fatty rats. A1: Normal retina in SD rats. A2: Higher magnification of A1. B1: Normal retina in SDT fatty rats. B2 Higher magnification of B1. C1: Shortening (arrow) and irregular arrangement (arrow heads) of cone and rod in retinal tissue in SDT fatty rats. C2: Higher magnification of $\mathrm{C} 1$. Bar $=200 \mathrm{~mm}$ in $\mathrm{A} 1 \mathrm{~B} 1$ and $\mathrm{C} 1,100 \mathrm{~mm}$ in $\mathrm{A} 2$, B2 and $\mathrm{C} 2$, respectively.

Epidemiological studies have elucidated several risk factors for cataracts including age, diabetes, and high body mass index [1,8]. In diabetes, severe metabolic disorders characterized by hyperglycemia and dyslipidemia can affect the eyes, and diabetic cataract is one of the earliest secondary complications of diabetes mellitus [20]. Some mechanisms, such as excessive sorbitol accumulation in tissue (via polyol pathway), abnormal glycosylation of lens protein (via non-enzyme glycation), and an increase of free radical production (oxidative stress), have been reported for cataract formation in diabetes [21-23]. Sustained hyperglycemia condition in SDT fatty rats induces the above mechanisms including acceleration of polyol pathway and/or increases of advanced glycation end products (AGEs) and reactive oxygen species (ROS), and as a result, it is considered that SDT fatty rats show severe cataracts (Table 2, Figure 1).

Surgical removal of the cataracts' lens is the only remedy, and pharmacological strategies for prevention of cataract are necessary. The anti-cataract agents classified in the following five categories: aldose reductase inhibitors, non-steroidal anti-inflammatory drugs, and agents acting on glutathione, antioxidant drugs, and miscellaneous drugs [23] In particular, the role of aldose reductase inhibitors for the prevention of diabetic cataract is well established in animal models [24,25].

Retinal folds in SDT fatty rats develop at an early age and peak at 24 weeks of age [26]. No obvious retinal folds were observed in this study. Perhaps only early retinal lesions have begun to appear, but it is possible that part of the process leading to retinal folds has been captured. A more detailed evaluation may be a part of elucidating the onset mechanism of DR. Also, SDT fatty rats may be a useful model for development of a new anticataract drug and the elucidation of the mechanism of progression of retinal lesions.

\section{CONCLUSION}

SDT fatty rat, a type 2 diabetes model of obesity, exhibit hyperglycemia and hyperlipidemia, and diabetic cataract characterized by macroscopic and microscopic changes made a progress with aging. SDT fatty rat may be useful in gaining a better understanding of the pathophysiology of cataracts and retinopathy as well as developing a new therapy for diabetic cataracts and retinopathy.

\section{REFERENCES}

[1] Gupta, S.K., Selvan, V.K., Agrawal, S.S., and Saxena, R. 2009, Advances in pharmacological strategies for the prevention of cataract development, Indian J. Ophthalmol. 57, 175-183.

[2] Heruye, S.H., Maffofou Nkenyi, L.N., Singh, N.U., Yalzadeh, D., Ngele, K.K., Njie-Mbye, Y.F., Ohia, S.E., and Opere, C.A. 2020, Current Trends in the Pharmacotherapy of Cataracts, Pharmaceuticals (Basel). 13.

[3] Calderon, G.D., Juarez, O.H., Hernandez, G.E., Punzo, S.M., and De la Cruz, Z.D. 2017, Oxidative stress and diabetic retinopathy: 
development and treatment, Eye (Lond). 31, 1122-1130.

[4] Khairallah, M., Kahloun, R., Bourne, R., Limburg, H., Flaxman, S.R., Jonas, J.B., Keeffe, J., Leasher, J., Naidoo, K., Pesudovs, K., et al. 2015, Number of people blind or visually impaired by cataract worldwide and in world regions, 1990 to 2010, Investig. Ophthalmol. Vis. Sci. 56, 6762-6769.

[5] Mitchell, P., Cumming, R.G., Attebo, K., and Panchapakesan, J. 1997, Prevalence of cataract in Australia: the Blue Mountains eye study, Ophthalmology 104, 581-588.

[6] Chua, J., Koh, J.Y., Tan, A.G., Zhao, W., Lamoureux, E., Mitchell, P., Wang, J.J., Wong, T.Y., and Cheng, C.-Y. 2015, Ancestry, Socioeconomic Status, and Age-Related Cataract in Asians: The Singapore Epidemiology of Eye Diseases Study, Ophthalmology 122, 2169-2178.

[7] Varma, R., and Torres, M. 2004, Prevalence of lens opacities in Latinos: the Los Angeles Latino Eye Study, Ophthalmology 111, 1449-1456.

[8] Thompson, J., and Lakhani, N. 2015, Cataracts, Prim. Care 42, 409-423.

[9] Galuppo, M., Giacoppo, S., Bramanti, P., and Mazzon, E. 2014, Use of natural compounds in the management of diabetic peripheral neuropathy, Molecules 19, 2877-2895.

[10] Leasher, J.L., Bourne, R.R.A., Flaxman, S.R., Jonas, J.B., Keeffe, J., Naidoo, K., Pesudovs, K., Price, H., White, R.A., Wong, T.Y., et al. 2016, Global Estimates on the Number of People Blind or Visually Impaired by Diabetic Retinopathy: A Meta-analysis From 1990 to 2010, Diabetes Care 39, 1643-1649.

[11] Mansour, S.E., Browning, D.J., Wong, K., Flynn, H.W.J., and Bhavsar, A.R. 2020, The Evolving Treatment of Diabetic Retinopathy, Clin. Ophthalmol. 14, 653-678.

[12] Masuyama, T., Katsuda, Y., and Shinohara, M. 2005, A novel model of obesity-related diabetes: introgression of the Lepr(fa) allele of the Zucker fatty rat into nonobese Spontaneously Diabetic Torii (SDT) rats, Exp Anim 54, 13-20.

[13] Ohta, T., Katsuda, Y., Miyajima, K., Sasase, T., Kimura, S., Tong, B., and Yamada, T. 2014, Gender differences in metabolic disorders and related diseases in Spontaneously Diabetic ToriiLepr(fa) rats, J Diabetes Res 2014, 841957..

[14] Matsui, K., Ohta, T., Oda, T., Sasase, T., Ueda, N., Miyajima, K., Masuyama, T., Shinohara, M., and Matsushita, M. 2008, Diabetes-associated complications in Spontaneously Diabetic Torii fatty rats, Exp Anim 57, 111-121.

[15] Katsuda, Y., Ohta, T., Miyajima, K., Kemmochi, Y., Sasase, T., Tong, B., Shinohara, M., and Yamada, T. 2014, Diabetic complications in obese type 2 diabetic rat models, Exp. Anim. 63, 121-32.

[16] Kemmochi, Y., Fukui, K., Maki, M., Kimura, S., Ishii, Y., Sasase, T., Miyajima, K., and Ohta, T. 2013, Metabolic Disorders and Diabetic Complications in Spontaneously Diabetic Torii Lepr (fa) Rat: A New Obese Type 2 Diabetic Model, J. Diabetes Res. 2013, 948257.

[17] Yamaguchi, T., Sasase, T., Mera, Y., Tomimoto, D., Tadaki, H., Kemmochi, Y., Ohta, T., Sato, E., and Matsushita, M. 2012, Diabetic peripheral neuropathy in Spontaneously Diabetic ToriiLepr(fa) (SDT fatty) rats, J Vet Med Sci 74, 16691673.

[18] Katsuda, Y., Sasase, T., Tadaki, H., Mera, Y., Motohashi, Y., Kemmochi, Y., Toyoda, K., Kakimoto, K., Kume, S., and Ohta, T. 2015, Contribution of hyperglycemia on diabetic complications in obese type 2 diabetic SDT fatty rats: effects of SGLT inhibitor phlorizin, Exp Anim 64, 161-169.

[19] Maekawa, T., Tadaki, H., Sasase, T., Motohashi, Y., Miyajima, K., Ohta, T., and Kume, S. 2017, Pathophysiological profiles of SDT fatty rats, a potential new diabetic peripheral neuropathy model, J Pharmacol Toxicol Methods 88, 160 166.

[20] Kyselova, Z., Stefek, M., and Bauer, V. 2004, Pharmacological prevention of diabetic cataract, J. Diabetes Complications 18, 129-140.

[21] Steers, W.D., Ciambotti, J., Etzel, B., Erdman, S., and de Groat, W.C. 1991, Alterations in afferent pathways from the urinary bladder of the rat in response to partial urethral obstruction, J. Comp. Neurol. 310, 401-410.

[22] Rowan, S., Bejarano, E., and Taylor, A. 2018, BBA - Molecular Basis of Disease Mechanistic targeting of advanced glycation end-products in age-related diseases, BBA - Mol. Basis Dis. 1864, 3631-3643.

[23] Aksoy, H., Keles, S., Koçer, I., and Akçay, F. 2001, Diabetic cataract and the total antioxidant status in aqueous humor, Clin. Chem. Lab. Med. 39, 143-145.

[24] Stribling, D., Mirrlees, D.J., Harrison, H.E., and Earl, D.C. 1985, Properties of ICI 128,436, a novel aldose reductase inhibitor, and its effects on diabetic complications in the rat, Metabolism. 34, 336-344.

[25] Zenon, G.J. 3rd, Abobo, C. V, Carter, B.L., and Ball, D.W. 1990, Potential use of aldose reductase inhibitors to prevent diabetic complications, Clin. Pharm. 9, 446-457.

[26] Tanaka, Y., Takagi, R., Ohta, T., Sasase, T., Kobayashi, M., Toyoda, F., Shimmura, M., Kinoshita, N., Takano, H., and Kakehashi, A. 2019, Pathological Features of Diabetic 
Retinopathy in Spontaneously Diabetic Torii

Fatty Rats, J. Diabetes Res. 2019. 